\title{
Online drawings for dementia diagnose: in-air and pressure information analysis
}

\author{
Marcos Faundez-Zanuy ${ }^{1}$, Enric Sesa-Nogueras ${ }^{1}$, Josep Roure-Alcobé ${ }^{1}$, Josep Garré-Olmo ${ }^{2}$, Karmele \\ Lopez-de-Ipiña ${ }^{3}$, Jordi Solé-Casals ${ }^{4}$ \\ ${ }^{1}$ Escola Universitària Politècnica de Mataró (UPC), Tecnocampus 08302 Mataró, Spain \\ faundez@eupmt.es \\ ${ }^{2}$ Institut d'assistència sanitària, Salt (Girona, Spain) \\ ${ }^{3}$ UPV/EHU (Basque Country University) Donostia \\ ${ }^{4}$ Digital Technologies Group. University of Vic
}

\begin{abstract}
In this paper we present experimental results comparing on-line drawings for control population (left and right hand) as well as Alzheimer disease patients. The drawings have been acquired by means of a digitizing tablet, which acquires time information angles and pressures. Experimental measures based on pressure and in-air movements appear to be significantly different for both groups, even when control population performs the tasks with the non-dominant hand.
\end{abstract}

Keywords- Nonlinear Speech Processing, Alzheimer disease diagnosis, Spontaneous Speech, Fractal Dimensions.

\section{INTRODUCTION}

It is well established the use of handwritten tasks for dementia diagnose. In the past, the analysis of handwriting had to be performed in an offline manner. Only the writing itself (strokes on a paper) were available for analysis. Nowadays, modern capturing devices, such as digitizing tablets and pens (with or without ink) can gather data without losing its temporal dimension. When spatiotemporal information is available, its analysis is referred as online. Modern digitizing tablets not only gather the $\mathrm{x}-\mathrm{y}$ coordinates that describe the movement of the writing device as it changes its position, but it can also collect other data, mainly the pressure exerted by the writing device on the writing surface and also the azimuth, the angle of the pen in the horizontal plane, and the altitude, the angle of the pen with respect the vertical axis (fig. 1 and 2). A very interesting aspect of the modern online analysis of handwriting is that it can take into account information collected when the writing device was not exerting pressure on the writing surface. Thus, the movements performed by the hand while writing a text can be split into two classes:

(a) On-surface trajectories (pen-downs), corresponding to the movements executed while the writing device is touching the writing surface. Each of these trajectories produces a visible stroke.

(b) In-air trajectories (pen-ups), corresponding to the movements performed by the hand while transitioning from one stroke to the next. During these movements the writing device exerts no pressure on the surface.

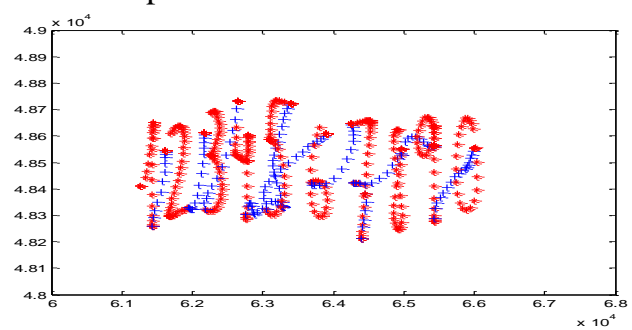

Figure 1. Example of handwritten numerical digits input onto a digitizing tablet. Asterisks $(*)$ represent pen-down information and cross $(+)$ the pen-up.

Fig. 1 shows the acquisition of the ten digits from 1 to 0 using an Intuos Wacom 4 digitizing tablet. The tablet acquired 100 samples per second including the spatial coordinates $(\mathrm{x}, \mathrm{y})$, the pressure, and a couple of angles (see Fig. 2). The pen-up in-formation is represented in Fig 1 using "+" while the pen- down is marked with "*". Our experiments on the biometric recognition of people reveal that these two kinds of information are complementary [1] and in fact, contain a similar discriminative capability, even when using a database of 370 users [2-3].

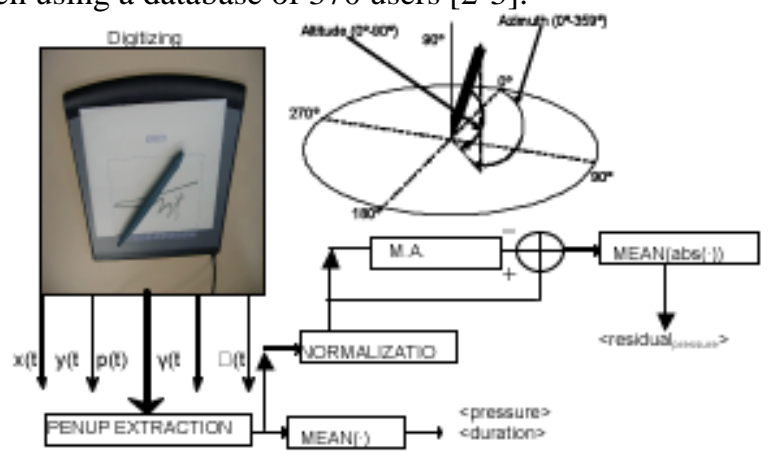

Figure 2. Information extracted from the digitizing tablet and pressure extracted measures. 


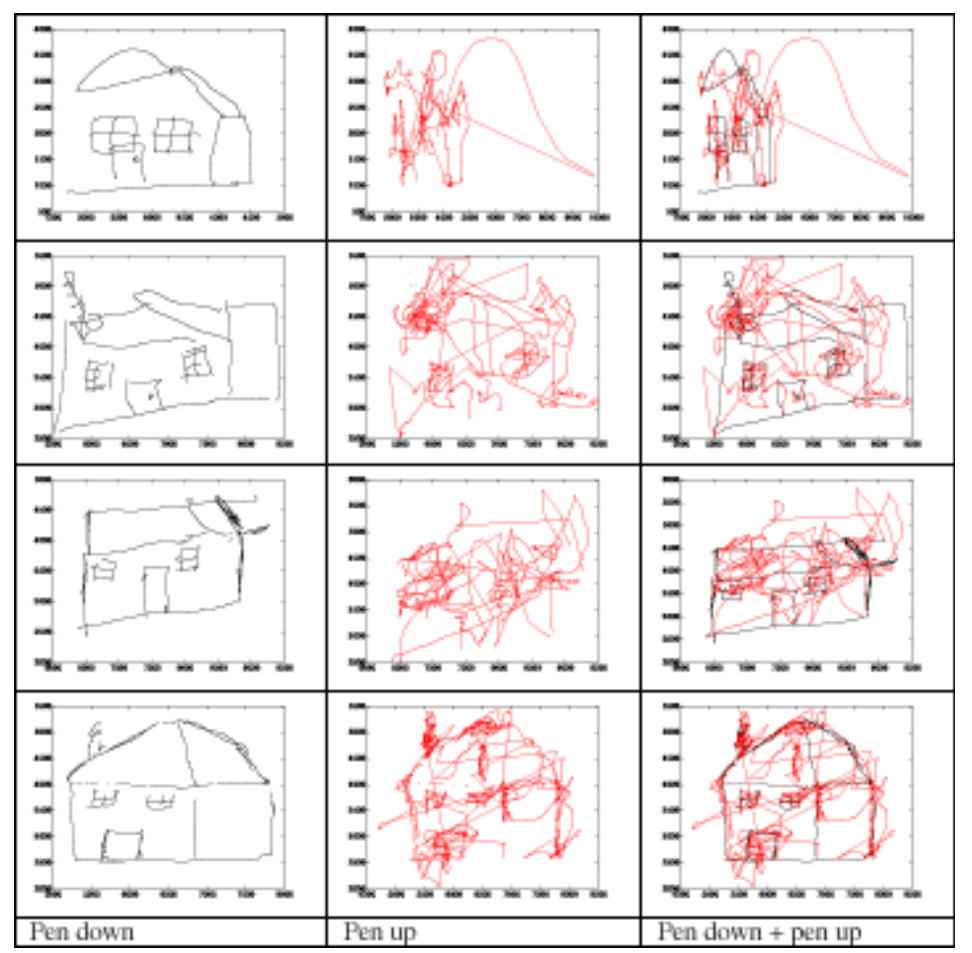

Figure 3. House drawing performed by four individuals with Alzheimer's disease (one per row). Each column corresponds to pen-down, pen-up and both simultaneously

\section{ON-LINE DRAWING APPLIED TO HEALTH ANALYSIS}

In the medical field, the study of handwriting has proven to be an aid to diagnose and track some diseases of the nervous system. For instance, handwriting skill degradation and Alzheimer's disease (AD) appear to be significantly correlated [4] and some handwriting aspects can be good indicators for its diagnosis [5] or help differentiate between mild Alzheimer's disease and mild cognitive impairment [6]. Also, the analysis of handwriting has proven useful to assess the effects of substances such alcohol [7] [8], marijuana [9] or caffeine [10]. Aided by modern acquisition devices, the field of psychology has also benefitted from the analysis of handwriting. For instance in [11], Rosenblum et al. link the proficiency of the writers to the length of the in-air trajectories of their handwritings. In the Figure 3 we present one complex drawing with three dimensions performed by individuals with AD of different clinical severity. The visual inspection of the pen down image suggest a progressive degree of impairment, where drawing becomes more disorganized and the three dimensions effect is only achieved in the mild case. The visual information provided by the pen up drawing between AD individuals also indicates a progressive impairment and disorganization when the individuals try to plan the drawing. It is also important to note that the comparison of the pen-up drawing between the mild case of $\mathrm{AD}$ and the control (Figures 4) also shows important differences. Besides the increased time on air, there is an increased number of hand movements before decide to put the pen in the surface to drawn. We consider that these graphomotor measures applied to the analysis of drawing and writing functions may be a useful alternative to study the precise nature and progression of the drawing and writing disorders associated with several neurodegenerative diseases. In addition, the pressure is also different. AD people produce softer and simpler strokes. Figure 5 corresponds to the pressure profiles of $\mathrm{AD}$ people, figure 6 to control group using the dominant hand and figure 8 to the nondominant hand of the control group. In the next section we propose several pressure related measures.

\subsection{Pressure derived measures}

One important advantage of online drawing is the possibility to evaluate the handwriting pressure from a quantitative point of view. In this paper, we will use the averaged pressure as well as a new proposed measure which consists of the mean absolute value of the residual signal obtained by the difference between the original pressure signal and a smoothed version obtained by a simple moving averaging filter. 


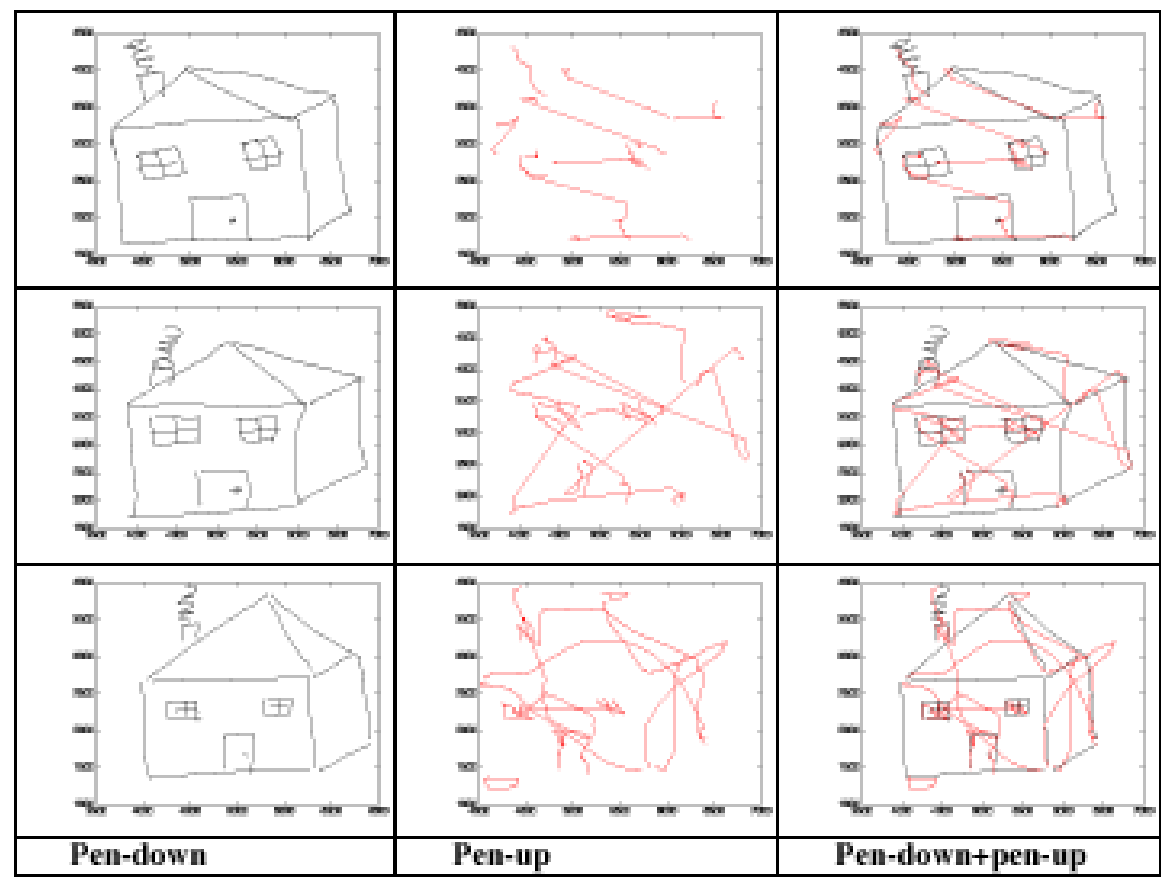

Figure 4. House drawing performed by three control people (one per row). Each column corresponds to pen-down, pen-up and both simultaneously, performed with the dominant hand.

In our experiments, the moving averaging filter consists of averaging 5 consecutive equally weighted samples. Figure 2 represents the diagram of blocks of this algorithm. Figure 9 shows on the top a portion of a sample pressure signal, the output of the averaging filter and the residual signal between both signals on the bottom. The simplest the pressure movements, the smallest the residual signals are (the easiest to predict the signal). Looking at the experimental results of table 1 it is evident the higher time for in-air movements for the AD group, which are around 7 times longer. On the contrary, the time on surface is just around 3 times longer. Thus, there are more differences between control and $\mathrm{AD}$ groups when looking at in-air movements. When comparing the non-dominant movements performed by the control group we obtained a 5.6 ratio and 1.5. Again, the in-air times are significantly higher for the $\mathrm{AD}$ group than the control group. Looking at the pressures, we observe that the mean pressure is 2.4 times higher for control group and dominant hand, and 2.1 for the non-dominant hand. About the mean absolute value of the residual error, the ratios are respectively 2.2 and 1.3 . thus, we can conclude that control group with non-dominant hand are in a middle situation between control group dominant hand and $\mathrm{AD}$ group.
Table 1. Statistical analysis/descriptives from the drawings shown in figures 4,5 and 6 .

\begin{tabular}{llll}
\hline Measurement & \multicolumn{2}{c}{ control } & pathological \\
\hline Dominant & $\begin{array}{c}\text { Non- } \\
\text { hominant } \\
\text { hand }\end{array}$ & $\begin{array}{c}\text { Dominant } \\
\text { hand }\end{array}$ \\
\hline Time in-air & 8334 & 10927 & 61008 \\
Time on-surface & 9680 & 22177 & 31521 \\
Total time & 18014 & 33104 & 92259 \\
$\begin{array}{l}\text { <Pressure> } \\
\text { <abs(Residual }\end{array}$ & 1307.8 & 1144.4 & 545.01 \\
error)> & 22.49 & 12.89 & 10.27 \\
\hline
\end{tabular}

\section{EXPERIMENTAL RESULTS}

In this paper we have evaluated the following measures:

a) Time related measures: time in air, time on-surface, and total time (in-air plus on-surface times). The time has been measured as the number of samples.

b) Pressure measures: mean pressure on-surface and mean absolute value of the residual signal between the onsurface pressure and the moving averaged pressure.

Table 1 summarizes some experimental measures of the drawings shown in figures 4, 5 and 6 . 

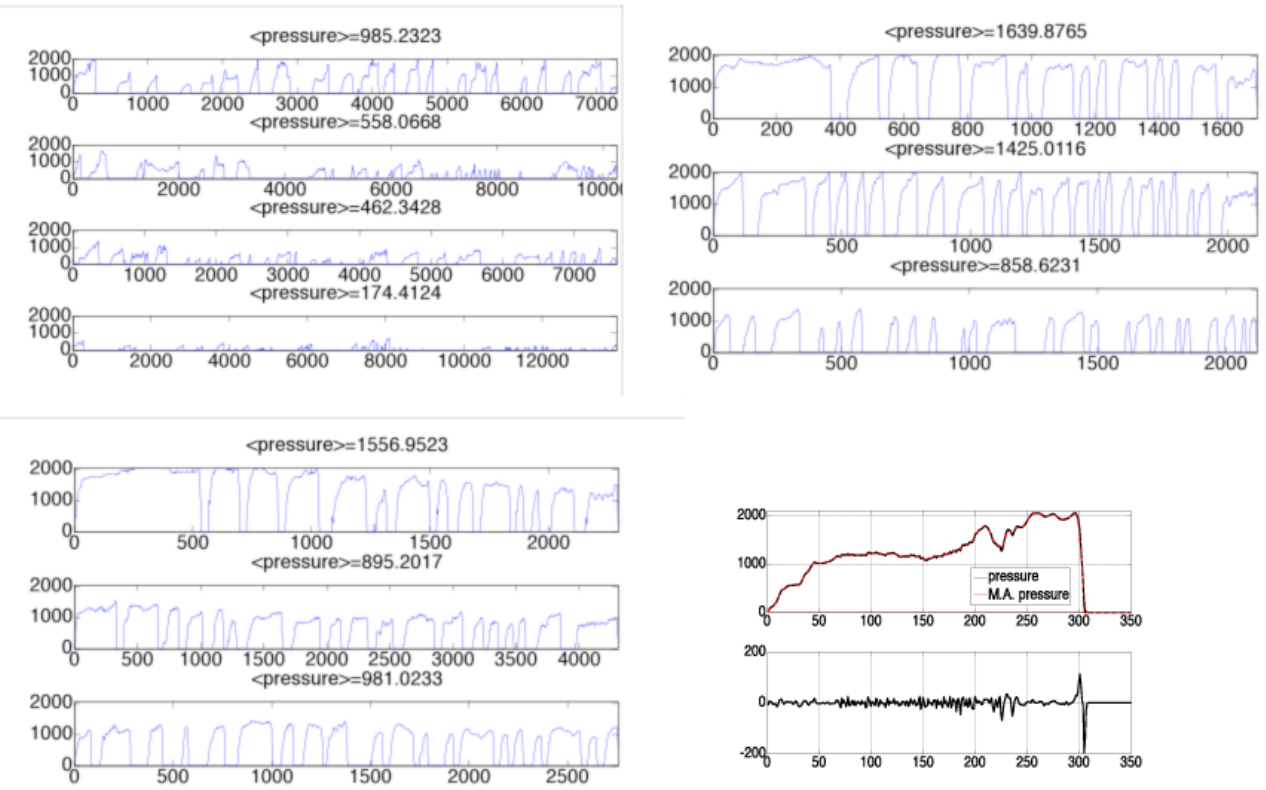

Figure 5. (a) Pressure profiles for three AD patients, as well as the average pressure (<pressure>), (b) pressure profiles samples for control group, dominant hand, as well as the average pressure, (c) pressure profiles samples for control group, non-dominant hand, as well as the average pressure and (d) a sample of pressure information and the moving average prediction on the top. The difference between both signals on the bottom.

\section{CONCLUSIONS}

Although some pathological drawings may look "normal" according to pen-down information, the pen-up information and the pressure look quite entangled and should permit easier diagnose. This observation points out the convenience of online handwriting analysis, which can outperform the classic offline mode, mainly due to the larger amount of available information, as well as the more accurate measurements made by the computer. The differences between control and pathological group do not seem to be related to some physical problem, because the control group, even when using the non-dominant hand performs less entangled pen-up movements. Future work will include some other measurements, with a larger database [12].

\section{ACKNOWLEDGMENT}

This work has been supported by FEDER and MEC, TEC2009-14123-C04-04, COST-2102 and SAIOTEK.

\section{REFERENCES}

1. E. Sesa-Nogueras, M. Faundez-Zanuy and Jiri Mekyska "An information analysis of in-air and on-surface trajectories in online handwriting" Cognitive Computation. DOI 10.1007/s12559-011-9119-y

2. E. Sesa-Nogueras and M. Faundez-Zanuy "Biometric recognition using online uppercase handwritten text" Pattern Recognition Vol. 45 (2012) pp 128-144. January 2012
3. E. Sesa-Nogueras and M. Faundez-Zanuy "Writer recognition enhancement by means of synthetically generated handwritten text". Engineering applications of artificial intelligence. In press. Doi: 10.1016/j.engappai.2012.03.010

4. KE Forbes, MF Shanks, A Venneri. The evolution of dysgraphia in Alzheimer's disease, Brain Res.Bull. 63 (2004) 19-24.

5. J Neils-Strunjas, K Groves-Wright, P Mashima, S Harnish. Dysgraphia in Alzheimer's disease: a review for clinical and research purposes, J.Speech Lang.Hear.Res. 49 (2006) 1313-1330.

6. P Werner, S Rosenblum, G Bar-On, J Heinik, A Korczyn. Handwriting process variables discriminating mild Alzheimer's disease and mild cognitive impairment, J.Gerontol.B Psychol.Sci.Soc.Sci. 61 (2006) p228-36.

7. F Asicioglu, N Turan. Handwriting changes under the effect of alcohol, Forensic Sci.Int. 132 (2003) 201-210.

8. JG Phillips, RP Ogeil, F Müller. Alcohol consumption and handwriting: A kinematic analysis, Human Movement Science. 28 (2009) 619632.

9. RG Foley, A Lamar Miller. The effects of marijuana and alcohol usage on handwriting, Forensic Sci.Int. 14 (1979) 159-164.

10. O Tucha, S Walitza, L Mecklinger, D Stasik, T Sontag, KW Lange. The effect of caffeine on handwriting movements in skilled writers, Human Movement Science. 25 (2006) 523-535.

11. S Rosenblum, S Parush, P Weiss. The in Air Phenomenon: Temporal and Spatial Correlates of the Handwriting Process, Perceptual \& Motor Skills. 96 (2003) 933.

12. 1. Faundez-Zanuy, M.; Hussain, A.; Mekyska, J.; Sesa-Nogueras, E.; Monte-Moreno, E.; Esposito, A.; Chetouani, M.; Garre-Olmo, J.; Abel, A.; Smekal, Z.; Lopez-de-Ipiña, K. Biometric Applications Related to Human Beings: There Is Life beyond Security. Cognitive Computation 2012, 5(1): 136-151. doi: 10.1007/s12559-012-9169-9. 